\title{
Factors Indicating the Accuracy of Fetal Weight Estimation in Preterm Newborns with Normal Amniotic Fluid Volume
}

\author{
Onur GURALP', Nevin TUTEN², Abdullah TUTEN², Altay GEZER ${ }^{3}$ \\ Oldenburg, Germany
}

\section{ABSTRACT}

OBJECTIVE: To determine the factors indicating the accuracy of fetal weight estimation in the last prenatal ultrasonography before delivery in preterm newborns with normal amniotic fluid volume.

STUDY DESIGN: Three hundred and seventy-one singleton pregnancies with normal amniotic fluid volume and delivered at between $24+0$ and $37+0$ gestational weeks were evaluated in a retrospective study. Any possible associations between the absolute percentage error of fetal weight estimations and the maternal and fetal data were examined.

RESULTS: In 135 of the 371 women (36\%), the absolute percentage error was greater than $10 \%$. The mean absolute percentage error was $8.7 \pm 7.5 \%$. The mean absolute percentage errors were $4.1 \pm 2.7 \%$ and $16.8 \pm 6.4 \%$ in the accurate and inaccurate estimation groups, respectively.

The rate of women examined during labor was significantly higher in the inaccurate estimation group compared to the accurate estimation group. There were no significant differences in age, body-massindex, gestational age at delivery, estimated-fetal-weight, actual birth weight, days from the last ultrasound examination to delivery, small for gestational age rate, placenta localization or fetal presentation between the two groups.

Examination during labor $(\beta=0.224)$ was the most important factor for the prediction of the absolute percentage error, followed by gestational age at delivery $(\beta=-0.198)$, presence of $(\beta=-0.158)$, and bodymass-index $(\beta=0.142)$.

CONCLUSION: In $36 \%$ of our study population, the absolute percentage error was $>10 \%$. Examination during labor was the most important factor for the prediction of the absolute percentage error, followed by gestational age at delivery, presence of small for gestational age, and body-mass-index.

Keywords: Absolute percentage error, Estimated, Fetal weight, Inaccuracy rate, Prediction of birth weight, Preterm newborn

\section{Gynecol Obstet Reprod Med 2021;27(1):17-21}

${ }^{1}$ Carl von Ossietzky Oldenburg University, University Hospital of Obstetrics and Gynecology, Klinikum Oldenburg, AöR, Oldenburg, Germany

${ }^{2}$ Kanuni Sultan Suleyman Education and Research Hospital, Obstetrics and Gynecology Istanbul, Turkey

${ }^{3}$ Istanbul Cerrahpasa University, Department of Obstetrics and Gynecology, Istanbul, Turkey

Address of Correspondence: Onur Guralp

University Hospital of Obstetrics and Gynecology, Klinikum Oldenburg, AöR Carl von Ossietzky Oldenburg University, Rahel-Strauß-Straße 10, 26133, Oldenburg, Germany

onur.gueralp@uni-oldenburg.de

Submitted for Publication: 24.07.2019 Revised for Publication: 26.09.2019 Accepted for Publication: 12.04 .2020

ORCID IDs of the authors:

OG:0000-0002-3517-3046, NT:0000-0001-8609-4770

AT: 0000-0002-8495-6426, AG:0000-0003-4884-7780

\begin{tabular}{|c|c|}
\hline \multirow{3}{*}{ 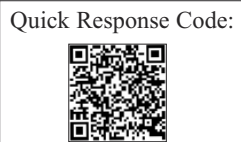 } & Access this article online \\
\hline & $\begin{array}{l}\text { Website: www.gorm.com.tr } \\
\text { e- mail: info@gorm.com.tr }\end{array}$ \\
\hline & DOI:10.21613/GORM.2019.1012 \\
\hline
\end{tabular}

How to cite this article: Guralp O. Tuten N. Tuten A. Gezer A. Factors Indicating the Accuracy of Fetal Weight Estimation in Preterm Newborns with Normal Amniotic Fluid Volume. Gynecol Obstet Reprod Med 2021;27 (1): 17-21

\section{Introduction}

Fetal weight estimation is a function of various biometric parameters and is calculated by applying those biometric parameters to various formulas (1). It is important to make an accurate estimation since the fetal weight informs about the fetal development and potential decision regarding delivery timing and even method. The comparison of two consecutive ultrasound examinations with an approximately 7 to 10-day interval is important to assess fetal weight gain and development. The fetal weight during the initial examination may be described as appropriate for gestational age (AGA), macrosomia or growth retardation. The comparison of the first and second examinations shows a slowing, arrest or development according to the respective growth percentile. An arrest of growth may be interpreted as an indication for delivery depending on the pregnancy week, doppler findings or fetal lung maturity state. A very low birth weight with a cervical opening may be an indication for a cesarean section, especially in multiple gestations (2). For those reasons, it is crucial to make an accurate fetal weight estimation. 
Numerous variables such as race, the experience of the sonographer, the quality of the ultrasound machine and probe, fetal malformations, gestational age, amniotic fluid volume, and maternal body mass index (BMI) may influence the accuracy of the fetal weight estimation (1-4). Some studies show that fetal gender, multiple gestation, fetal presentation, and placental location may also play a role $(4,5)$.

Most of the studies on fetal weight estimation were performed in term pregnancies and the formulas to calculate fetal weight are mainly suitable for this group of pregnancies $(2,3)$. In other words, there are fewer studies on preterm pregnancies regarding the rate of the accuracy of fetal weight estimation and the factors affecting it. At this point, in order to interpret the clinical relevance of the accuracy of fetal weight estimation, it is important to know the factors which may affect the accuracy of fetal weight estimation in various subgroups of pregnancies.

In our study, we aim to determine the factors indicating the accuracy of fetal weight estimation in the last prenatal ultrasonography before delivery in preterm pregnancies with normal amniotic fluid volume.

\section{Material and Method}

All singleton pregnancies delivered between $24+0$ gestational weeks $(\mathrm{GW})$ and $37+0 \mathrm{GW}$ in the Obstetrics and Gynecology Department of Istanbul Cerrahpasa University, School of Medicine between 2008 and 2015 were investigated in a retrospective study. The study was approved by the Ethics Committee of the Istanbul Cerrahpasa University (06.11.2014, 02-235911). Consent for using data was obtained from the patients. The study was conducted in accordance with the Declaration of Helsinki.

The inclusion criteria were singleton pregnancies with normal amniotic fluid volume and delivery between $24+0$ and $37+0 \mathrm{GW}$.

The exclusion criteria were congenital anomalies, multiple gestations, and a duration of more than 7 days between ultrasound examination and delivery.

Maternal and fetal demographic data (maternal age, BMI), clinical data (gestational age (GA) at delivery, presence of preterm rupture of membranes, the status of initiation of labor), and ultrasonographic measurements and findings in several weeks prior to delivery (biparietal diameter (BPD), head circumference (HC), abdominal circumference (AC), femur length (FL), estimated fetal weight (EFW), amniotic fluid index, fetal presentation, and placental insertion site) were recorded.

Ultrasonographic examinations were performed by obstetrics residents with similar experience with the Sonoscape SSI 5000 Ultrasound (China). The Hadlock I formula ( $\log 10$ weight $=1.35960 .00386 \mathrm{AC} 9 \mathrm{FL}+0.0064 \mathrm{HC}+0.00061 \mathrm{BPD}$ $9 \mathrm{AC}+0.0424 \mathrm{AC}+0.174 \mathrm{FL}$ ) was used to calculate the fetal weight.

The GA was calculated according to the last menstrual period using Naegele's formula. The GA according to the last menstrual period was checked by comparing it with the first trimester crown-rump length (CRL) measurement. A difference of more than 7 days was accepted as significant and the GA was corrected according to the first-trimester CRL measurement.

Weight at birth between the $10^{\text {th }}$ and $90^{\text {th }}$ percentiles according to the pregnancy week was defined as AGA, $<10^{\text {th }}$ percentile was defined as SGA, and $>90^{\text {th }}$ percentile was defined as LGA.

The absolute percentage error was calculated as:

( the actual birth weight - estimated fetal weight / the actual birth weight $) \times 100$.

The study group was divided into two groups, namely the accurate and inaccurate estimation groups. The accurate and inaccurate estimation groups included the cases with absolute deviation rates of $<10 \%$ and $\geq 10 \%$, respectively.

All the parameters in the groups were compared and the possible association between these parameters and the normal inaccuracy rate was investigated.

\section{Statistical analysis}

All analyses were performed using the Statistical Package for the Social Sciences (SPSS) software version 18.0 (SPSS Inc., Chicago, IL). The Kolmogorov- Smirnov test was used to assess the normality of the distribution of the variables. The homogeneous parametric variables were presented as mean \pm standard deviation (SD). The categoric variables were presented as percentages (\%). The comparison between the groups was performed using either an independent samples ttest or a Chi-square test depending on the variable. A linear regression analysis was performed to evaluate the factors which may influence the absolute percentage error. A $p$-value of $<0.05$ was accepted as statistically significant.

\section{Results}

A total of 371 women were included in the statistical evaluation. In 135 women $(36 \%)$, the absolute percentage error was $>10 \%$.

Considering all women in the study, the mean absolute percentage error was $8.7 \pm 7.5 \%$. The mean absolute percentage errors were $4.1 \pm 2.7 \%$ and $16.8 \pm 6.4 \%$ in the accurate and inaccurate estimation groups, respectively.

The demographical, clinical, and sonographic parameters of the accurate and inaccurate estimation groups are shown in table I. 
Table I: Comparison of the accurate and inaccurate estimation groups

\begin{tabular}{|c|c|c|c|c|}
\hline & $\begin{array}{l}\text { Accurate Estimation } \\
\qquad(n=236)\end{array}$ & $\begin{array}{l}\text { Inaccurate Estimation } \\
\qquad(n=135)\end{array}$ & $p$ & Test \\
\hline Age (y) & $29.7 \pm 6.4$ & $30.3 \pm 5.7$ & 0.400 & $\mathrm{~T}$ \\
\hline BMI $\left(\mathrm{kg} / \mathrm{m}^{2}\right)$ & $27.95 \pm 4.92$ & $28.79 \pm 5.03$ & 0.117 & $\mathrm{~T}$ \\
\hline GA at delivery $(w)$ & $32.50 \pm 3.08$ & $31.91 \pm 3.53$ & 0.109 & $\mathrm{~T}$ \\
\hline EFW (g) & $1749 \pm 554$ & $1734 \pm 638$ & 0.806 & $\mathrm{~T}$ \\
\hline Weight at birth (g) & $1738 \pm 542$ & $1630 \pm 567$ & 0.070 & $\mathrm{~T}$ \\
\hline Days from USG to delivery (d) & $1.12 \pm 1.89$ & $0.96 \pm 1.80$ & 0.427 & $\mathrm{~T}$ \\
\hline SGA-Rate & $43.2 \%$ & $48.9 \%$ & 0.291 & $\chi^{2}$ \\
\hline Anterior wall placenta rate & $40.3 \%$ & $45.9 \%$ & 0.294 & $\chi^{2}$ \\
\hline Placenta praevia rate & $8.1 \%$ & $7.4 \%$ & 0.809 & $\chi^{2}$ \\
\hline \multicolumn{5}{|l|}{ Fetal presentation rates } \\
\hline Head & $76.3 \%$ & $66.7 \%$ & 0.125 & $\chi^{2}$ \\
\hline Breech & $19.9 \%$ & $28.9 \%$ & & \\
\hline Transverse & $3.8 \%$ & $4.4 \%$ & & \\
\hline Rate of women examined during labor & $25 \%$ & $39.3 \%$ & 0.004 & $\chi^{2}$ \\
\hline
\end{tabular}

BMI: Body mass index, GA: Gestational age, EFW: Estimated fetal weight, SGA: Small for gestational age $p<0.05$ (bold) is significant. T: T-Test, $\chi$ :Chi-square test

There was no significant difference in age, BMI, GA at delivery, EFW, weight at birth, days from USG to delivery (within 7 days), SGA rate, placenta localization or fetal presentation between the accurate and inaccurate estimation groups.

The rate of women examined during labor was significantly higher in the inaccurate estimation group compared to the accurate estimation group. The mean absolute percentage error rate was $10.5 \pm 6.9 \%$ in the examination during labor group and $5.9 \pm 7.7 \%$ in the examination not-during-labor group $(p=0.001)$.

The linear regression analysis with the dependent variable absolute percentage error and independent variables BMI, GA at delivery, placental location, fetal presentation, examination during labor, duration between examination to delivery within 7 days, and presence of SGA showed that BMI ( $p=0.006)$, GA at delivery $(p<0.001)$, examination during labor $(p<0.001)$, and presence of SGA $(p=0.004)$ were significant for the prediction of the absolute percentage error (Table II). Placental location, fetal presentation, and duration between examination and delivery within 7 days were not significant.

Standardized coefficients $(\beta)$ showed that examination during labor $(\beta=0.224)$ was the most important factor for the prediction of the absolute percentage error, followed by GA at delivery $(\beta=-0.198)$, presence of SGA $(\beta=0.158)$, and BMI $(\beta=0.142)$.

\section{Discussion}

In our study on preterm pregnancies with normal amniotic fluid volume, the mean absolute percentage error was $8.7 \pm 7.5 \%$. In $36 \%$ of the women, the absolute percentage error was $>10 \%$. Examination during labor was the only significant parameter between accurate and inaccurate estimation groups.

Considering all women in the study, the linear regression analysis showed that examination during labor, GA at delivery, presence of SGA, and BMI were significant for the pre-

Table II: Linear regression analysis for the prediction of absolute inaccurate measurement

\begin{tabular}{lccrc}
\hline & & & \multicolumn{2}{c}{ Confidence interval } \\
\cline { 3 - 5 } & $p$ & $B$ & Lower bound & Upper bound \\
\hline BMI & $\mathbf{0 . 0 0 6}$ & 0.216 & 0.061 & 0.370 \\
GA at delivery & $<0.001$ & -0.461 & -0.715 & -0.207 \\
Placental location & 0.525 & 0.228 & -0.476 & -1.002 \\
Fetal presentation & 0.569 & 0.409 & 1.993 & 1.819 \\
Examination during labor & $<\mathbf{0 . 0 0 1}$ & 3.688 & -0.433 & 0.383 \\
Duration between examination to delivery & 0.887 & 0.029 & 0.755 & 4.060 \\
Presence of SGA & $\mathbf{0 . 0 0 4}$ & 2.407 &
\end{tabular}

B: Unstandardized regressions coefficient, BMI: Body mass index, GA: Gestational age, SGA: Small for gestational age $p<0.05$ (bold) is significant. 
diction of the absolute percentage error in preterm pregnancies with normal amniotic fluid volume in descending order of weight, whereas placental location, fetal presentation, and examination during labor and duration between examination and delivery within 7 days were not significant.

Gestational age plays an important role in neonatal survival in preterm pregnancies (1). Berger et al. showed that fetal weight, prophylaxis for respiratory distress syndrome, single or multiple birth, and fetal gender were found to have an impact on neonatal outcomes with between 22 and 26 completed weeks of gestation (6). Singleton female newborns who received antenatal corticosteroid therapy had a higher birth weight, and were treated in intensive care were found to have significantly better outcomes (7). At this point, the accuracy of fetal weight estimation and the recognition of the factors which may influence the accuracy are essential to making decisions concerning obstetrics management as well as informing the pregnant women about the deviation rates of fetal weight estimation.

The role of fetal weight, pregnancy week, maternal BMI, amniotic fluid index, fetal presentation, fetal gender, placental location, and number of fetuses and the influence of the experience of the physician have been evaluated in many studies as possible confounding factors affecting the accuracy of fetal weight estimation in preterm pregnancies (8-15). There is a scarcity of data on the impact of active labor in the preterm pregnancy subgroup.

Hadlock 1 is one of the most commonly used formulas for fetal weight estimation, and it was also used in our study. Kurmanavicius et al. (2) compared the success rates of fetal weight estimation using Hadlock formulas in the fetal weight groups between $500 \mathrm{~g}$ and $5000 \mathrm{~g}$ and detected that the Hadlock formulas showed the most stable results in all of the weight groups. In their study, the deviation rate was $6.2 \%$ in term pregnancies.

Siemer et al. evaluated 160 women with a birth weight $<2500$ g for estimated fetal weight with the Hadlock 1 formula and detected an absolute percentage error of $8.90 \% \pm 6.92 \%$, which is comparable to our results (3). In most publications on estimated fetal weight accuracy, the patients were evaluated according to the weight groups rather than the appropriateness of birth weight according to pregnancy week. In our study, we also evaluated SGA and AGA cases in preterm pregnancies; the presence of SGA was associated with an unstandardized coefficient of 2.4, which means a mean deviation or error of $24 \mathrm{~g}$ (95 CI: 7-40 g) in a $1000 \mathrm{~g}$ fetus.

The role of fetal presentation in fetal weight estimation is controversial. Many of the authors have detected no influence (8-11), whereas a few others found that a breech presentation was associated with a higher error percentage $(12,13)$. In our study, the presentation had no significant effect on the accuracy of fetal weight estimation.
Faschingbauer et al. evaluated the influence of active labor on fetal weight estimation in term pregnancies and found that active labor was associated with a higher error percentage (14). In our study, the presence of active labor had a significant influence on the accuracy of fetal weight estimation with an unstandardized coefficient of 3.6, which means a mean deviation or error of $36 \mathrm{~g}$ (95 CI: $19-53 \mathrm{~g}$ ) in a $1000 \mathrm{~g}$ fetus. The presence of active labor may influence the evaluation of the head of the fetus, which is more likely to descend into the pelvis, potentially making it difficult to get the optimal plane for the BPD and $\mathrm{HC}$ measurements. Moreover, frequent painful contractions, the posture changes of the patient during the examination, and the hastiness of the physician in such a constellation may theoretically play a role in affecting the accuracy of the fetal weight estimation.

The influence of placental location on fetal weight estimation has been evaluated in many studies and it does not seem to have any effect $(8,15,16)$, as our findings also indicate.

In our study, BMI had a significant influence on fetal weight estimation in the linear regression analysis, albeit with a very low unstandardized coefficient of 0.2 , which means a mean deviation or error of $2 \mathrm{~g}$ per $\mathrm{kg} / \mathrm{m}^{2}$ change in BMI in a $1000 \mathrm{~g}$ fetus. In other words, a BMI change of 10 units $\left(\mathrm{kg} / \mathrm{m}^{2}\right)$ means a $20 \mathrm{~g}$ error for a $1000 \mathrm{~g}$ fetus. This may be statistically significant but is clinically less relevant. Many other studies have concluded that BMI does not seem to have an effect on the accuracy of fetal weight estimation $(8,10,15-18)$. It is difficult to interpret the results of BMI in a population of pregnant women with a great range of GA, from $24+0 \mathrm{GW}$ to $37+0$ GW. BMI changes throughout the pregnancy as the baby grows; moreover, the amount of amniotic fluid and maternal blood volume, as well as the presence and extent of edemas, may also influence the BMI value. The thickness of the fat tissue around the abdomen may theoretically influence the visualization quality, however, BMI does not always reflect only the amount of fat tissue around the abdomen.

In our study design, the limit for the time interval between the last ultrasound examination and delivery was set at a maximum of 7 days. However, the mean interval was considerably shorter than the upper limit, with a mean of approximately 24 hours. The linear regression analysis showed that the time interval did not have a significant effect on the accuracy of fetal weight estimation. Studies on the effect of the time interval between the last ultrasound examination and delivery on the accuracy of the fetal weight estimation have yielded inconsistent results. Kaaij et al. set the maximum time interval between the last fetal weight estimation and delivery at 14 days with a mean time interval of 4.1 days and did not detect any effect (19). Scott et al. set the same maximum time interval of 14 days, had a mean time interval of 3.8 days and found that the number of days between the last fetal weight estimation and delivery was significantly associated with a high error rate (15).

The main limitation of our study is its retrospective nature 
and relatively small sample size. With a prospective study, the parameters can be better standardized among each other so that a comparison of individual parameters is possible. Even though our study included a greater number of patients than many other comparable studies, in order to compare the effect of various parameters, an even greater number of women is obviously better for the clinical relevance as well as statistical power.

If we consider a $10 \%$ error rate as clinically significant, then the results of this study can be better interpreted. Although the linear regression analysis showed that examination during labor, GA at delivery, presence of SGA, and BMI were significant for the prediction of the absolute deviation rate, only "examination during labor" had a significant effect on the inaccurate estimation group compared to the accurate estimation group.

\section{Conclusion}

The absolute percentage error was $>10 \%$ in $36 \%$ of the preterm pregnancies with normal amniotic fluid volume. Examination during labor was the most important factor for the prediction of the absolute percentage error, followed by GA at delivery, presence of SGA, and BMI in descending order; therefore, these factors should be considered in an interpretation of the accuracy of fetal weight estimation. Placental location, fetal presentation, and duration between examination to delivery within 7 days did not have a significant effect on fetal weight estimation.

Acknowledgments: None

Funding Statement: None

Disclosures: The authors report no conflicts of interest

Author Contributions: OG., Concept, Data Processing, Analysis, and Interpretation, Writer. NT. Data Collection, Processing, Analysis, and Interpretation. AT. Supervision, Concept, Design, Data Collection and Processing, Statistical analysis and Data Interpretation. AG. Supervision, Concept, Design, Analysis and Interpretation

\section{References}

1. Dudley NJ. A systematic review of the ultrasound estimation of fetal weight. Ultrasound Obstet Gynecol. 2005; 25(1):80-9. doi: 10.1002/uog.1751.

2. Kurmanavicius J. Burkhardt T. Wisser J. Huch R. Ultrasonographic fetal weight estimation: accuracy of formulas and accuracy of examiners by birth weight from 500 to 5000 g. J Perinat Med. 2004;32(2):155-61. doi: 10.1515/JPM.2004.028.

3. Siemer J. Egger N. Hart N. Meurer B. Müller A. Dathe O. et al. Fetal weight estimation by ultrasound: comparison of 11 different formulae and examiners with differing skill levels. Ultraschall Med. 2008;29(2):159-64. doi: 10.1515/ JPM.2004.028.

4. Huber C. Zdanowicz JA. Mueller M. Surbek D. Factors influencing the accuracy of fetal weight estimation with a focus on preterm birth at the limit of viability: a systematic literature review. Fetal Diagn Ther. 2014;36(1):1-8. doi: $10.1159 / 000358518$.

5. Melamed N. Yogev Y. Ben-Haroush A. Meizner I. Mashiach R. Glezerman M. Does use of a sex-specific model improve the accuracy of sonographic weight estimation? Ultrasound Obstet Gynecol. 2012;39(5):549-57. doi: 10.1002/uog.10064.

6. Berger TM. Bernet V. El Alama S. Fauchere JC. Hösli I. Irion O. et al. Perinatal care at the limit of viability between 22 and 26 completed weeks of gestation in Switzerland. 2011 revision of the Swiss recommendations. Swiss Med Wkly. 2011;141:w13280. doi: 10.4414/ smw.2011.13280.

7. Tyson JE. Parikh NA. Langer J. Green C. Higgins RD. National Institute of Child Health and Human Development Neonatal Research Network. Intensive care for extreme prematurity - moving beyond gestational age. N Engl J Med. 2008;358(16):1672-81. doi:10.1056/ NEJMoa073059.

8. Heer IM. Kumper C. Vogtle N. Muller-Egloff S. Dugas M. Strauss A. Analysis of factors influencing the ultrasonic fetal weight estimation. Fetal Diagn Ther. 2008;23 (3):204-10. doi: 10.1159/000116742.

9. Mills MD. Nageotte MP. Elliott JP. Crade M. Dorchester W. Reliability of ultrasonographic formulary in the prediction of fetal weight and survival of very-low-birthweight infants. Am J Obstet Gynecol 1990;163(5 Pt 1): 1568-74. doi: 10.1016/0002-9378(90)90629-1.

10. Edwards A. Goff J. Baker L. Accuracy and modifying factors of the sonographic estimation of fetal weight in a high-risk population. Aust N Z J Obstet Gynaecol. 2001; 41(2):187-90. doi: 10.1111/j.1479-828x.2001.tb01206.x.

11. Ragosch V. Entezami M. Hundertmark S. Mutz A. Becker R. Weitzel H. Accuracy of ultrasound weight assessment-comparison of vertex vs. breech presentation. Ultraschall Med. 1997;18(1):19-25. doi: 10.1055/s-2007-1000510.

12. Melamed N. Ben-Haroush A. Meizner I. Mashiach R. Glezerman M. Yogev Y. Accuracy of sonographic weight estimation as a function of fetal sex. Ultrasound Obstet Gynecol. 2011;38(1):67-73. doi: 10.1002/uog.8914.

13. Chauhan SP. Magann EF. Naef RW $3^{\text {rd }}$. Martin JN Jr. Morrison JC. Sonographic assessment of birth weight among breech presentations. Ultrasound Obstet Gynecol. 1995;6(1):54-7.doi:10.1046/j.1469-0705.1995. 06010054.x

14. Faschingbauer F. Dammer U. Raabe E. Schneider M. Faschingbauer C. Schmid M. et al. Intrapartum sonographic weight estimation. Arch Gynecol Obstet. 2015; 292(4):805-11. doi: 10.1007/s00404-015-3720-3.

15. Scott F. Beeby P. Abbott J. Accuracy of estimated fetal weight below 1,000 g. Aust N Z J Obstet Gynaecol. 1996; 36(2):129-32. doi: 10.1111/j.1479-828x.1996.tb03267.x. 
16. Shamley KT. Landon MB. Accuracy and modifying factors for ultrasonographic determination of fetal weight at term. Obstet Gynecol. 1994;84(6):926-30.

17. Field NT. Piper JM. Langer O. The effect of maternal obesity on the accuracy of fetal weight estimation. Obstet Gynecol. 1995;86(1):102-7. doi: 10.1016/0029-7844(95) 00096-A.
18. Farrell T1. Holmes R. Stone P. The effect of body mass index on three methods of fetal weight estimation. BJOG. 2002;109(6):651-7. doi: 10.1111/j.14710528.2002.01249.x.

19. Kaaij MW. Struijk PC. Lotgering FK. Accuracy of sonographic estimates of fetal weight in very small infants. Ultrasound Obstet Gynecol. 1999;13(2):99-102. doi: 10.1046/j.1469-0705.1999.13020099.x. 